الطباقية الحياتية للفورامنيفرا الطافية لتكوين شيرانش في بئر مخمور (1) منطقة مخمور شمال العرلق

$$
\begin{aligned}
& \text { عبد الله سلطان شهاب الحديدي } \\
& \text { قسم علوم الأرض التطبيقية } \\
& \text { كلية العلوم } \\
& \text { جامعة تكريت }
\end{aligned}
$$

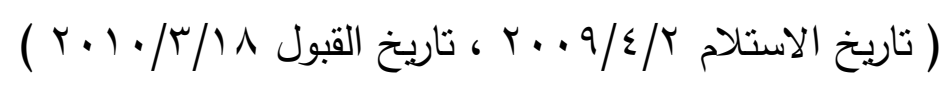

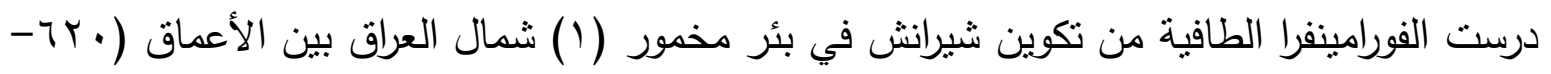

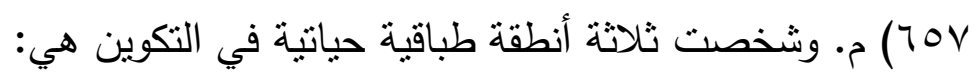

1. Globotruncana elevata-Rosita fornicata Zone.

2. Globotruncana aegyptiaca Zone.

3. Gansserina gansseri Zone.

قورنت نتائج الدراسة الحالية مع دراسات سابقة داخل القطر وخارجه وحدد في البئر المذكور عمر تكوين شيرانش من الكامبانيان المتأخر الى الماسترختيان المبكر .

\title{
Planktonic Foraminiferal Biostratigrapy of Shiranish Formation in Makhmur Well No. (1), Makhmur Area, Northern Iraq
}

\author{
Abdullah S. Sh. Al-Hadiedi \\ Department of Applied Geology \\ College of Science \\ University of Tikrit
}

\begin{abstract}
Planktonic foraminifera is studied from Shiranish Formation in Makhmur well No. (1), North Iraq between the depth interval (657-620) $\mathrm{m}$. Three biostratigraphy Zones are recognized in these formation:

1. Globotruncanita elevata-Rosita fornicata Zone.

2. Globotruncana aegyptiaca Zone.

3. Gansserina gansseri Zone.
\end{abstract}


The results of this study are compared with the work of others inside and outside Iraq. The age of the Shiranish Formation in the said well is Late Companian to Early Maastrichtian.

\section{المقدمة}

وصف هذا التكوين لأول مرة من قبل (Henson, 1940, In: Bellen et al., 1959) في نطاق الطيات العالية في شمال العراق قرب قرية شيرانش إسلام شمال شرق زاخو يتألف التكوين من المارل والحجر الجيري المارلي التي تمنل الترسيب في البيئات البحرية العميقة المفتوحة وبعمر يمتد من الكامبانيان المتأخر إلى عمر الماسترختيان (Buday, 1980) • يتطرق البحث الحالي لأول مرة إلى دراسة الطباقية الحياتية للفورامنيفرا الطافية في نكوين شيرانش في بئر مخمور (1) الذي يقع في حقل مخمور النفطي في منطقة

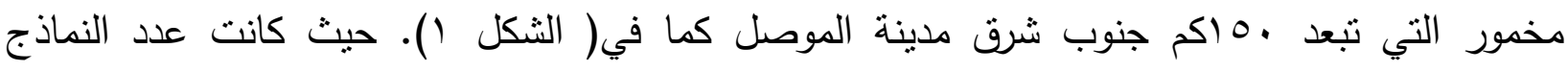

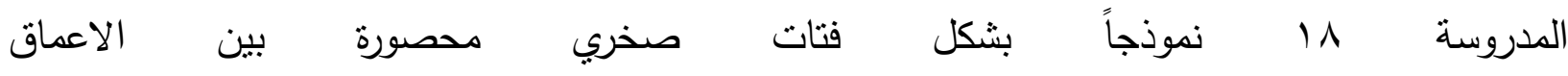
(657-620) م. حيث إن النموذجين ا ور11 حسب المعلومات المأخوذة من شركة نفط الثمال بمثلان

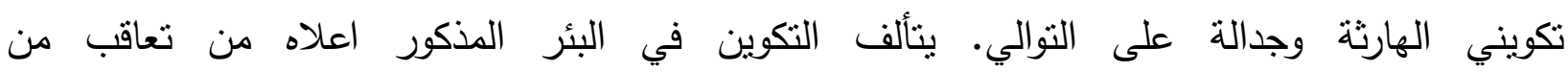
المارل والحجر الجيري المارلي والحجر الجيري الطيني، الحدود السفلى له مع تكوين هارثة منوافقة أما الحدود العليا مع تكوين جدالة فغير متوافقة. درس (Kassab, 1973) تكوين شيرانش في مقطعه النموذجي وحدد عمرُ بالماسترختيان، كما درس (Kassab et al., 1986) هذا التكوين في بئر ساسان (1) شمال غرب العراق وحددا عمرهُ بالماسترختيان المبكر إلى الأوسط ، كما درس (Ghafor, 1988) تكوين شيرانش في بئر حجر -1 منطقة سنجار، شمال غرب العراق وحدد عمره بأعلى الماسترختيان، كما درس( Bakkal et al., 1993 نكوين شيرانش في منطقة هجران

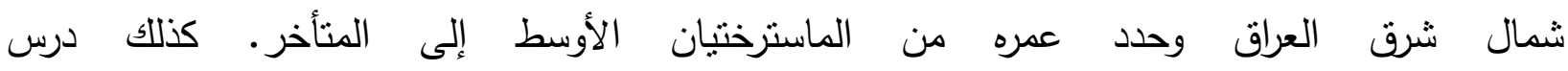
(Al-Mutwali, 1996) المتأخر الى أسفل الماسترختيان المتأخر ، كذلك درست (Hammoudi, 2000) هذا التكوين في بئر مخمور (TI) وحددت عمره بالكامبانيان المنأخر الى الماسترختيان المنتوسط ـ كما درس كل من تكوين شيرانش في منطقة (AL-Jubouri, 2002 and Al-Mutwali) , ( Al-Jubouri 2005) سنجار شمال غرب العراق وحددوا عمرة من الكامبانيان المتأخر الى الماسترختيان المتأخر ، ودرس (Sharbazheri, 2007) تكوين شيرانش في منطقة سماكول شمال شرق العراق وحدد عمره من 
الكامبانيان المتأخر الى الماسترختيان المبكر، كذلك درس (Sharbazheri, 2008) تكوين شيرانش في منطقة السليمانية شمال شرق العراق وحدد عمره بالماسترختيان المبكر الى الماسترختيان المتأخر.

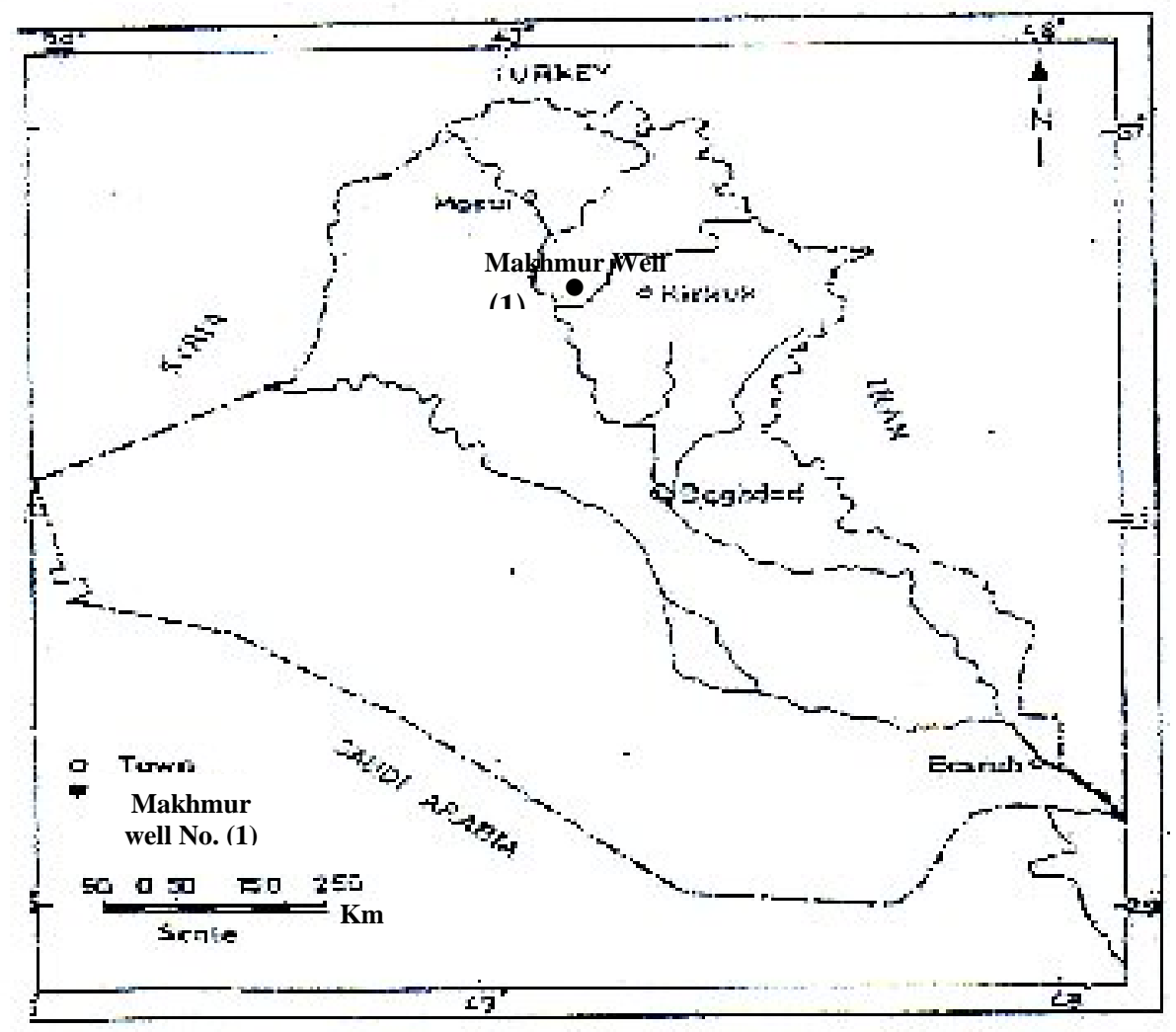

الثكل 1: خارطة منطقة الدراسة

اعتماداً على التوزيع الطباقي والانتشار النسبي لحشود الفورامنيفرا الطافية تم تقسيم تكوين شيرانش في منطقة الدراسة الى ثلاثة انطقة حياتية كما في(الثكل 2). نم مضاهاة الأنطقة الحالية مع الأنطقة المشخصة من قبل الدراسات السابقة داخل وخارج القطر كما في (الثكل ؟ب). تبين أن النطاق الأول والثاني يقعان ضمن الكامبانيان المنأخر في حين النطاق الأخير يقع ضمن الكامبانيان المتأخر الى الماسترختيان المبكر • وفيما يأتي وصف الأنطقة من الأقدم الى الأحدث :

\section{Globotruncanita elevata-Rosita fornicata concurrent range Zone.}

تعريف النطاق: نطاق مدى منداخل Concurrent range Zone للنوعين الدالين على النطاق. حدود النطاق: يبدأ هذا النطاق مع ظهور كلا النوعين الدالين على النطاق وينتهي هذا النطاق بالظهور الأول للنوع Globotruncana aegyptiaca Nakkady

عمر النطاق: الكامبانيان المتأخر Late Campanian ع سمك النطاق: هذا النطاق بسمك (15) م بين الأعماق (642-657) م .

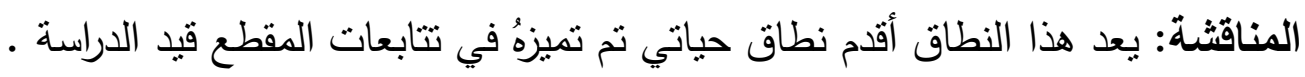




$$
\text { أهم الحشود الحياتية المميزة لهذا النطاق هي : }
$$

Globotruncana bulloides (Vogler), Glt. ventricosa White, Glt. mariei Banner and Blow, Glt. Rosetta (Carsey), Glt. orientalis El-Nagger, Glt. lapparenti Bolli, R. patelliformis (Gandolfi), R. plummerae (Gandolfi), Rugoglobigerina macrocephala (Bronnimann), Archaeoglobigerina cretacea (d'Orbigny).

المضاهاة: يضاهي النطاق الحالي الأنطقة Glt. calcarata Zone الموصوف من فبل (Bolli, 1966) (Caron, الموصوفة من قبل Glt. Calcarata Zone والتي تمنل الكامبانيان المتأخر ، كما يكافئ النطاق 1985 and Robaszunski et al., 1984)

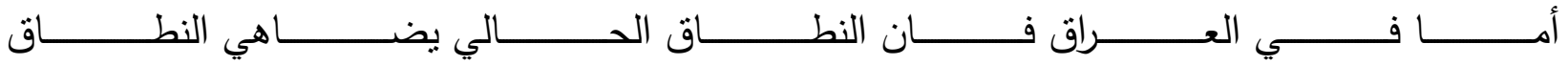
(Al-Mutwali, 1996) المشخص من قبل Glthartforms, Gltn. elevata, R. fornicata

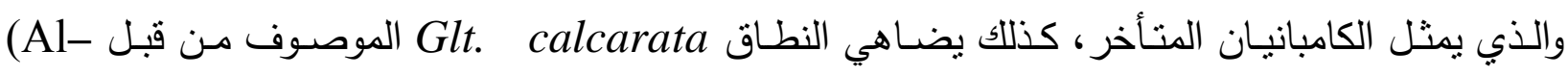
Mutwali and Al-Jubouri, 2005)

\section{Globotruncana aegyptiaca Interval Zone.}

تعريف النطاق: نطاق فاصل للنوع Globotruncana aegyptiaca

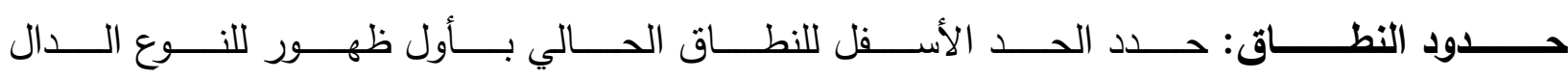
Globotruncana aegyptiaca - Gansserina gansseri عate Campanian عمر النطاق: الكامبانيان المتاخر سمك النطاق: سمك النطاق (15) م بين الأعماق (627-642) م المناقشة: يمتاز هذا النطاق بوفرة عالية لمجاميع الفورامنيفرا وأهم الحشود الحياتية المشخصة في هذا النطاق Globotruncana bulloides (Vogler), Globigerinelloides subcarinata Bronnimann, Rugoglobigerina macrocephala (Plummer), Rgl. rugosa (Plummer), Rosita fornicata (Plummer), Globotruncanita petteris (Gandolfi), Gln. elevata (Brotzen), Glt. lapparenti Bolli, Glt. orientalis El-Nagger, Glt. rosetta (Carsey), Glt. mariei Banner and Blow, Glt. ventricosa White.

المضاهاة: يضاهي النطاق الحالي من النطاق Glt. lapparenti tricarinata الموصوف من قبل

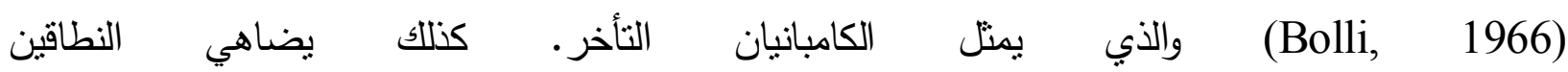
Glt. aegyptiaca Zone, Glnt.havanenis Zone 
والذي يمثل الكامبانيان المتأخر. أما في العراق (Caron, 1985 and Robaszynski, et al., 1984) يضاهي النطاق الحالي النطاق Glt. aegyptiaca الموصوف من قبل (Al-Mutwali, 1996) الذب يمثل

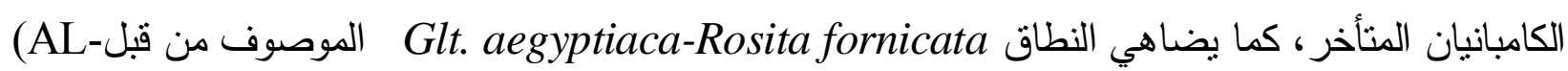
Mutwali and AL-Jubouri, 2005) Glt. aegyptiaca

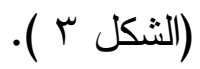

\section{Gansserina gansseri Interval Zone (part):}

تعريف النطاق: نطاق فاصل للنوع الدال Gansserina gansseri . حدود النطاق: حدد الحد الاسفل للنطاق بأول ظهور للنوع الدال Gansserina gansseri كما حدد الحد الأعلى للنطاق باختفاء الأنواع الدالة على النطاق. عمر النطاق: الكامبانيان المتاخر الى الماسترختيان المبكر Late Campanian -Early Maastrichtian سمك النطاق: سمك النطاق (7.0) م بين الأعماق (620-627). المناقثة: يمثل هذا النطاق الأخير في المقطع قبد الدراسة. أهم الحشود الحياتية المميزة لهذا النطاق هي : G. widenmayeri(Gandolfi), Rugoglobigerina macrocephala Bronnimann, Rosita contusa (Cushman), R. fornicata (Plummer), Globotruncanita pettersi (Gandolfi), Globotruncana duwi Nakkay, Glt. aegyptiaca Nakkay, Glt. lapparenti Bolli, Glt. orientalis El Naggar, Glt. rosetta (Carsey), Glt. mariei Banner and Blow.

المضاهاة: يضاهي النطاق الحالي النطاق Blt.gansseri الموصوف من قبل (Bolli.,1966) gansseri Gansserina والذي يمنل الماسترختيان المبكر، كذلك يكافئ أيضا النطاق المشخص من قبل (Caron,1985 and Robaszynski et al., 1984) والذي يمثل الماسترختيان المبكر ـ أما في العراق فان النطاق الحالي يضاهي النطاق الثانوي Glt. Gansseri gansseri الموصوف

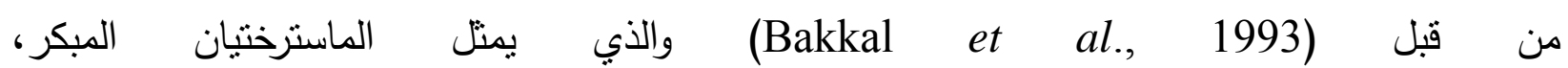

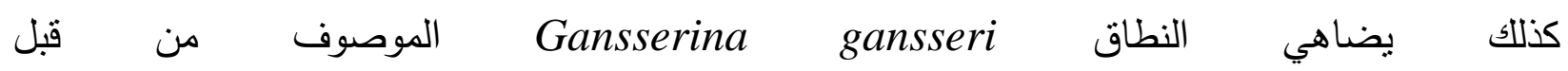
(AL-Mutwali, 1996, AL- Mutwali and Al - Jubouri, 2005 ) Contusotruncana contusa Zone ، Gansserina gansseri Zone المبكر ، كما يكافئ الأنطقة Pseudotextularia intermedia Zone، الماسترختيان المبكر كما في (الثكل r). 


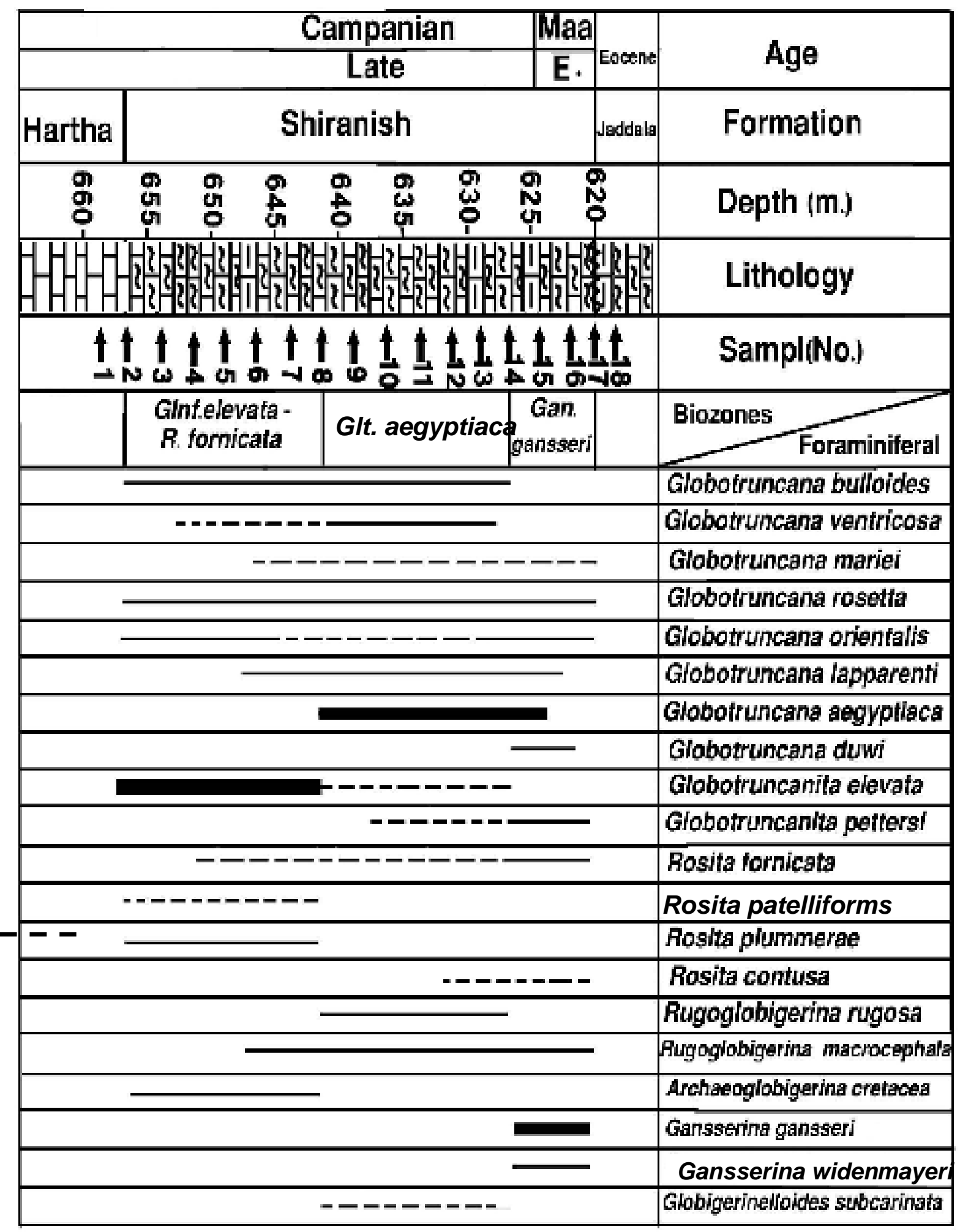

Abundant

Common

--- Rare

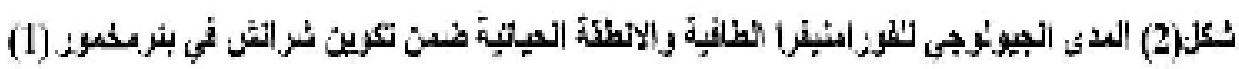


جدول r: معناهاة انطقه الفورامنيفرا الطافية مع الأنطقة المحدة في دراسات داخل وخارج القطر 


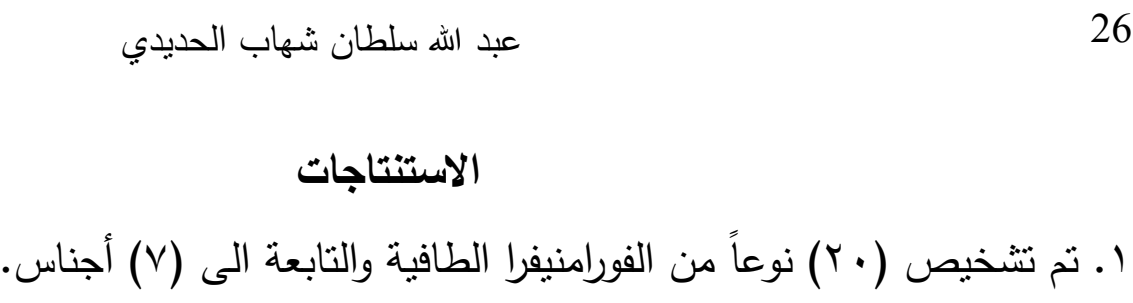

r. بناءا على طبيعة التوزبع الطباقي والانتشار النسبي للفورامنيفرا الطافية لتكوين شيرانش في بئر مخمور (1) تم تقيم المقطع قيد الدراسة الى الأنطقة الآتية : (1)

1. Globotruncanita elevata-Rosita fornicata Zone.

2. Globotruncana aegyptiaca Zone.

3. Gansserina gansseri Zone.

م. من مضاهاة الأنطقة الحالية مع الأنطقة الموصوفة داخل وخارج القطر تم تحديد عمر تكوين شيرانش في الدراسة الحالية من الكامبانيان المتأخر الى الماسترختيان المبكر .

\section{المصسادر الأجنبية}

Abawi, T.S., Abdel-Kireem, M.R. and Yousif, J.M., 1982. Planktonic foraminiferal Stratigraphy of Shiranish Formation, Sulaimaniah-Dokan Region Northeastern Iraq. Revista Espnola de Micropalentologia, Vol. 14, No.1, pp. 153-164.

Al-Jubouri, H.A., 2002. Planktic Foraminiferal Biostratigraphy and Depositional Environment of Shiranish Formation in Sinjar Area, North West Iraq. Unpub. M.Sc. Thesis, Mosul University.

Al-Mutwali, M.M., 1996. Planktonic Foraminiferal Biostratigraphy of the Shiranish Formation, Khashab Well No. 1, Hemeren Area, North Eastern Iraq. Jour. Geol. Sci. Iraq. Vol. 7, No. 1, pp. 129-136.

AL-Mutwali, M.M. and AL-Jubouri, F.N., 2005. Litho and Biostratigraphy of Shiranish Formation (Late Campanian-Maastrichtian) in Sinjar area. Northwestern Iraq. Rafidian Journal of Science, Vol. 16, No.1, Geology special issue. pp.152-176.

Bakkal, K.K. Ghafor ,I.M., and Kassab, I.I.M., 1993. Biostratigraphy of Shiranish Formation in Hijran Area Northeastern Iraq . Jour.of Science and Nature .Vol..2, No.2, pp. 34-39.

Bellen, R.C., Van; Dunnington, H.V.; Wetzel R., Morton, D., 1959. Lexique Stratigraphique International. Asie. Fasciule 10a, Iraq, Paris, 333 p.

Bolli, H.M., 1966. Zonation of Cretaceous to Policene Marine Sediments Based on Planktonic Foraminifera. Bul. Inform. Assoc. Venezuelan Geol. Min. Pet. Ina. Vol. 1, pp. 3-32. 
Buday, T.,1980. Regional Geology of Iraq. Vol.1 Stratigraphy ,I.I.M. Kassab and S. Z. Jassim (Eds) D. G. Geol. Surv. Min. invest. publ.445p.

Caron, M. 1985. Cretaceous Planktic Foraminifera. In: Bolli, H.M., Saunders, J.R. and Perch-Nielaem K. (eds.) Plankton Stratigraphy. Cambridge. Univ. Press, PP. 17-18, Figs. 37.

Ghafor , I.M. 1988. Planktonic Foraminifera and of the Aaliji Formation and Nature of its Contact with the Shiranish Formation in Well Tel-Hajer No.1, Sinjar area, Northeastern Iraq, M.Sc. Thesis (Unpub.) University of Salahddin, Iraq , 178 p. (in Arabic)

Hammoudi, R.A., 2000. Planktonic Foraminiferal Biostratigraphy of the Shiranish Formation (Upper Cretaceous) in Jambur well No. 13, Northern Iraq. Raf. Jour. Sci., Vol. 11, No. 4, PP. 50-58.

Kassab, I.I., 1973. Planktonic Foraminifera of the Shiranish Formation Type Locality, Northern Iraq. Jour. Geolo. Sic. Iraq. VI. PP. 100-109.

Kassab, I.I., Al-Omari, F.S. and A1-Safawee, N.A., 1986. The Cretaceous Tertiary Boundary in Iraq Represented by Subsurface Section of Sasan Well No. I., N.W. Iraq Jour. Geol. Sci. Iraq, Vol. 1, No. 2, PP. 129-167.

Sharbazheri, K. M., 2007. Planktonic Foraminiferal Biostratigraphy of the Upper Cretaceous Reddish Pale Brown Succession from Smaquli Area Northeastern Iraq (Kurdistan Region) Iraqi Bulletin of Geology and Mining - State Company of Geological Survey and Mining. Inpress.

Sharbazheri, K. M., 2008. Biostratigraphy and Paleoecology of Cretaceous/ Tertiary Boundary in the Sulaimani Region, Kurdistan, NE-Iraq. Unpub Ph. D Thesis, Sulaimani University. 


\section{Plate 1}

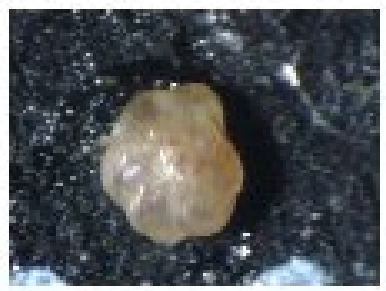

Gansserina gansseri

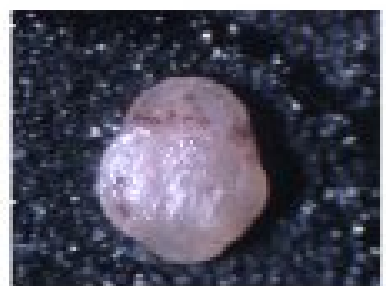

Globotruncana mariei

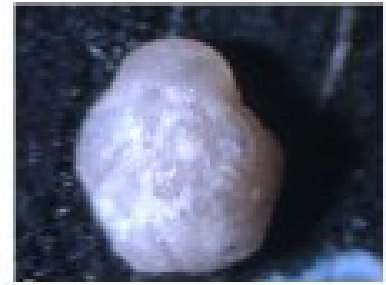

Globotruncana lapparenti

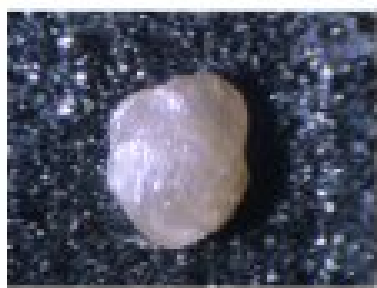

Globotruncana elevata

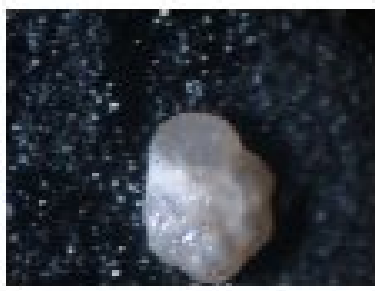

Gansserina gansseri

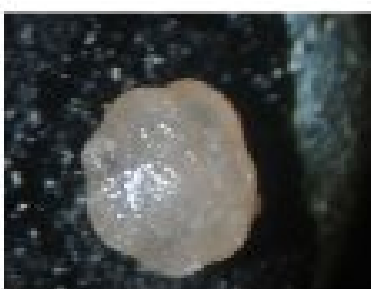

Globotrancana orientalis

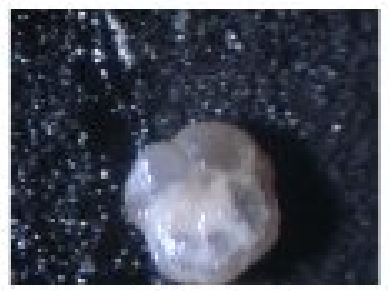

Globotrancana contusa

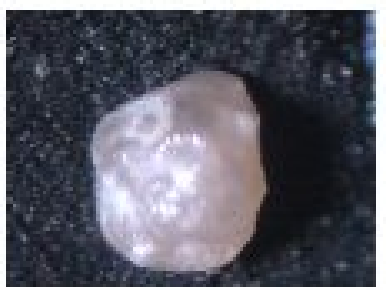

Rosita patelliformis

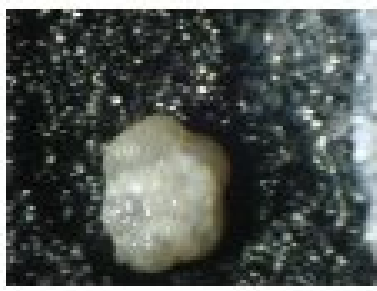

Rosita fornicata

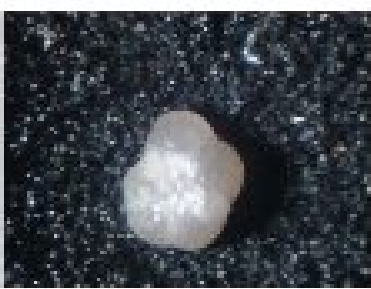

Globotrancana aegyptiaca

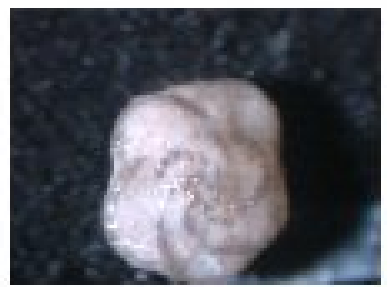

Globotrancana bulloides 